\title{
The study of the isolated power supply system operation with controlled distributed generation plants, energy storage units and drive load
}

\author{
$Y u N$ Bulatov $^{1}, A V$ Kryukov $^{2,3}$, and $K V$ Suslov $^{3, *}$ \\ ${ }^{1}$ Bratsk State University, 40, Makarenko st., Bratsk, Russia \\ ${ }^{2}$ Irkutsk State Transport University, 15, Chernyshevsky st., Irkutsk, Russia \\ ${ }^{3}$ Irkutsk National Research Technical University, 83, Lermontova st., Irkutsk, Russia
}

\begin{abstract}
Energy storage units (ESU) and distributed generation (DG) plants including those using renewable energy sources can be used to develop isolated power supply systems (IPSS) and enhance their reliability. Operation of DG plants in the IPSS requires to consider restrictions for consumers maximum load, as well as effects of abrupt load increase or load shedding on the generating plants. These problems can be resolved using ESU and intelligent technologies for DG plants control. The paper considers IPSS of an industrial facility with turbogenerator plants (TGP), wind power plant (WPP) and high-capacity ESU for which fuzzy control systems and prognostic controllers were used. The simulation was performed in the MATLAB system using the Simulink and SimPowerSystems software packages. Normal and emergency modes were studied in IPSS with TGP, WPP, and high-capacity ESU. The simulation results indicated that combined use of high-capacity ESU and prognostic controller of TGP generator rotor speed allows to ensure stability and survivability of IPSS enhancing its damping properties. The auto-prognostic controller of the TGP generator, which does not require special adjustment, provides high quality indicators of the IPSS control in all considered modes.
\end{abstract}

\section{Introduction}

A fairly large proportion of power supply systems operate in isolation, that is, without communication with a high-power electric energy system (EES). Energy storage units (ESU) [1-5] and distributed generation (DG) plants [6-8] including those using renewable energy sources [9-11], can be used to develop isolated power supply systems (IPSS) and enhance their reliability. When operating the DG plants in the IPSS, it is necessary to take into account the restrictions for consumers maximum load, as well as effects of IPSS operation dynamic changes on the generating plants. It is possible to smooth out and limit the possible fluctuations arising herewith and deviations of the controlled parameters by using high-power ESU and intelligent technologies to control DG plants [12-20].

As evidenced by studies, a fairly effective way to control DG plants based on synchronous generators is the use of fuzzy controllers [16,21-23] and prognostic algorithms [24-28], which make it possible to create adaptive systems. However, to implement practical application of such systems, laborious research is required using complex models, taking into account a large number of possible operating modes in order to determine their influence on the control parameters and quality indicators of the control process.
The work investigated an industrial facility IPSS with a drive load. The following elements were used as sources in the considered IPSS: a turbine generator plant (TGP) with an auto-prognostic generator rotor speed controller [218], a wind power plant (WPP) with a blade angle fuzzy controller [23] and a storage device, the energy intensity and power of which is sufficient to cover the facility entire load for a long time. The simulation was performed in the MATLAB system using the Simulink and SimPowerSystems software packages. Normal and emergency operating modes in IPSS are studied.

\section{Description of IPSS computer model with DG plant and ESU}

The diagram of the industrial facility IPSS under study, shown in Fig. 1, consisted of a $3125 \mathrm{kVA}$ TGPs, a highcapacity (ESU) and a 1 MVA wind power plant (WPP), connected together with a accumulator battery (AB) through a DC link and $0.4 / 10 \mathrm{kV}$ step-up transformer. Asynchronous motors (AM) were the main power consumers: two high-voltage AMs with a capacity of $670 \mathrm{~kW}$ each, as well as a large number of low-voltage AMs which are taken into account in the model in the form of equivalent units with a capacity of $930 \mathrm{~kW}$ and $1,485 \mathrm{~kW}$, respectively, powered from different $10 \mathrm{kV}$

\footnotetext{
* Corresponding author: souslov@istu.edu
} 
bus sections (Fig. 1). The consumers were energized by $10 \mathrm{kV}$ cable lines.

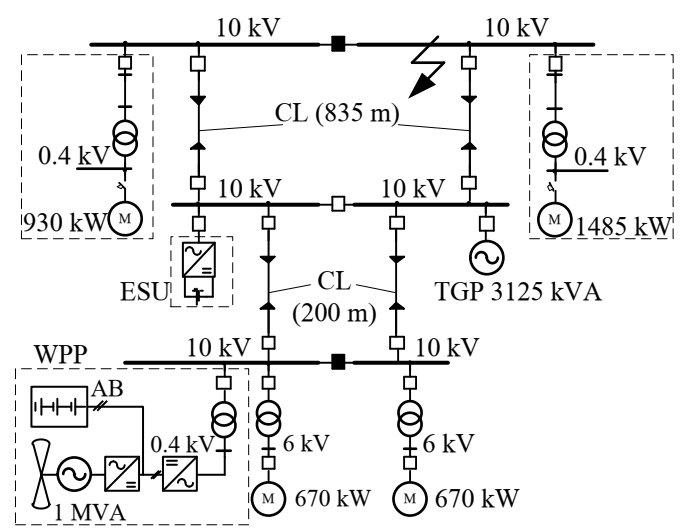

Fig. 1. The diagram of the industrial facility IPSS under study: $\mathrm{CL}$ - cable line.

The IPSS model under study was created in MATLAB environment while using Simulink and SimPowerSystems simulation modeling packages. The model of the used TGP steam turbine was described by the following differential equation:

$$
T_{\mathrm{T}} \cdot \frac{d P_{\mathrm{T}}}{d t}+P_{\mathrm{T}}=\mu
$$

where $P_{\mathrm{T}}$ - turbine power; $\mu$ - opening of a controlling element; $T_{\mathrm{T}}=0.2 \mathrm{~s}$ - turbine time constant.

The TGP generator excitation system was modeled using the first-order aperiodic link with transfer function $\frac{1}{0.025 s+1}$, and a series-connected amplifier with transfer function $\frac{1}{0.001 s+1}$, where $\mathrm{s}-$ Laplasian operator.

To stabilize the TGP generator parameters, the model used automatic excitation controllers (AEC) and speed controllers (ASC), which are proportional-integraldifferential (PID) controllers. Structural diagrams and the description of the used AEC and ASC models are presented in works $[15,16]$.

The diagram of the used Simulink-model of the auto- prognostic ASC is provided in Fig. 2, in which the time constant of the prognostic link was determined automatically depending on the synchronous generator load angle $\delta$ (the Forecast time unit in Fig. 2).

The use of ESU based on high-capacity accumulator batteries is a promising development line for use of IPSS. The simulation used a model of a lithium-ion accumulator battery represented by the Battery unit in the SimPowerSystems package of the MATLAB system.

A description of the WPP model with a fuzzy system to control the blades rotation angle is provided in [23]. A separate accumulator battery was simulated in WPP, when the battery was connected to the DC buses of the DC link, and used to stabilize the WPP parameters due to a change in wind speed.

\section{Simulation results}

The simulation was carried out in order to reveal the influence of a powerful energy storage unit and an autoprognostic controller of the rotor speed of the TGP main generator rotor on IPSS operating modes. The studies were carried out for the following operating modes of the IPSS under study with a powerful asynchronous load:

- short-time three-phase short circuit (SC);

- direct start of a powerful asynchronous motor;

- emergency WPP shutdown.

The initial TGP generator loading was $90 \%$; in this case, the modes with permanently connected or disconnected ESU were considered. When simulating a three-phase short circuit in the place indicated in Fig. 1, the complete scheme of the IPSS under study was considered and the comparison of the work of the classical and auto-prognostic ASC in terms of control quality indicators was performed. With a short circuit in the IPSS, a voltage dip occurred (Fig. 3), which, was not influenced by the auto-prognostic ASC as the simulation results indicated.
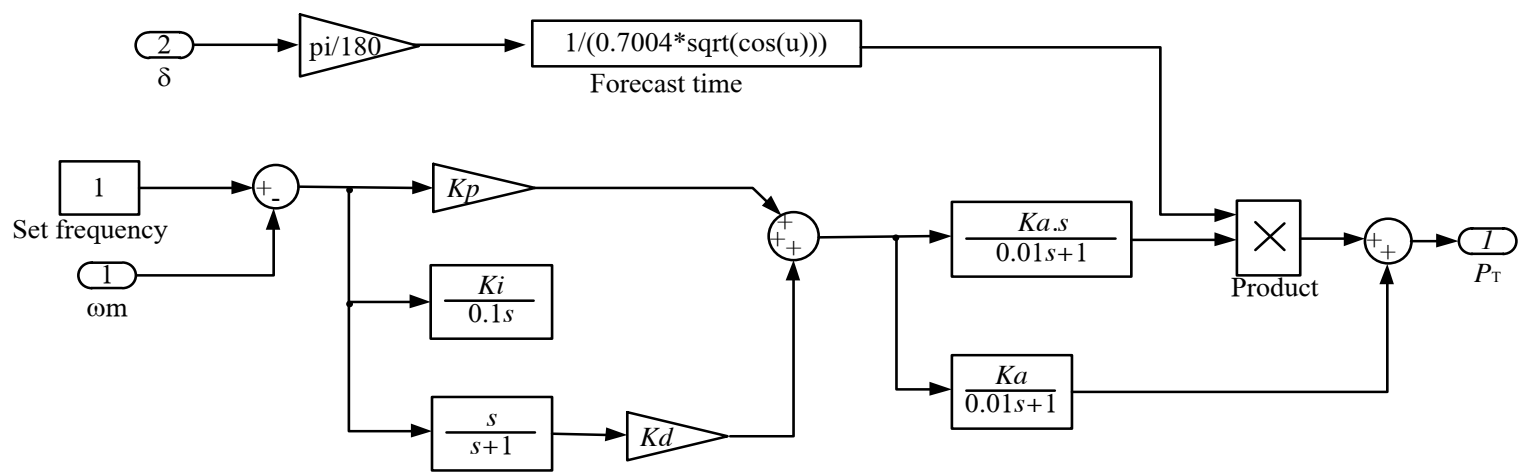

Fig. 2. Structural diagram of the auto-prognostic ASC model: $K p, K i, K d-$ ASC tuning coefficients. 


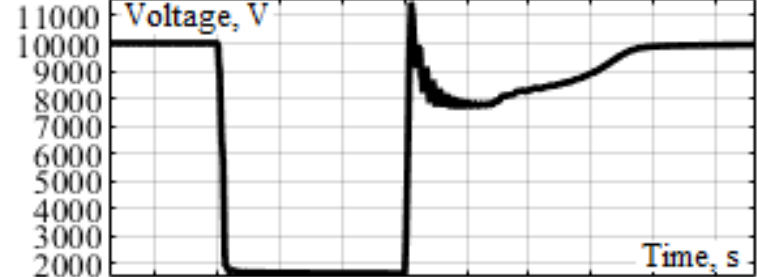

$\begin{array}{lllll}19.8 & 20 & 20.220 .420 .620 .8 & 21 & 21.221 .421 .6\end{array}$

Fig. 3. Time dependence of the effective voltage value in the IPSS for a short-term $(0.6 \mathrm{~s})$ three-phase short circuit.

The simulation results, presented in the form of time dependences of the turbine power and the generator rotor speed in Fig. 4, showed a significant influence of the

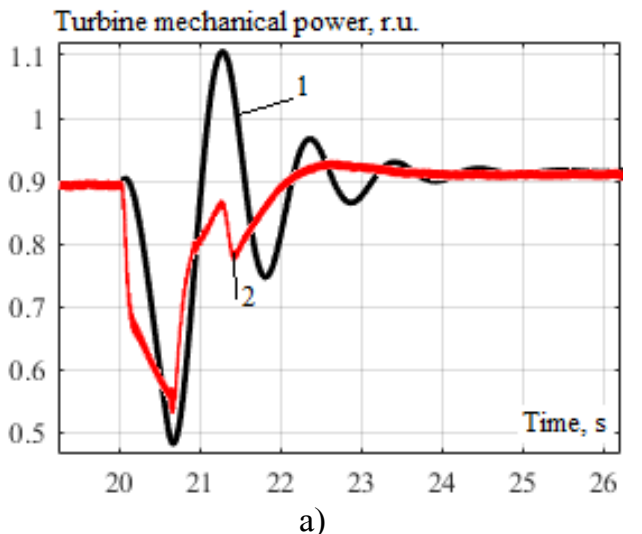

auto-prognostic ASC generator of the TGP on the control processes at a three-phase short circuit in the IPSS, making the turbine control more accurate and smooth; at the same time, the overshoot and oscillation of the mechanical power of the turbine significantly decreased in the case of using the auto-prognostic ASC (Fig. 4, a), the transient process time for the generator rotor speed decreased by 1.8 times, and the overshoot value for the same parameter was reduced by 9 times Fig. 4, b).

The simulation results also showed an increase in short-circuit currents when using all sources, including a high-power ESU, which significantly feeds the shortcircuit location (Fig. 5). Thus, the use of powerful ESU

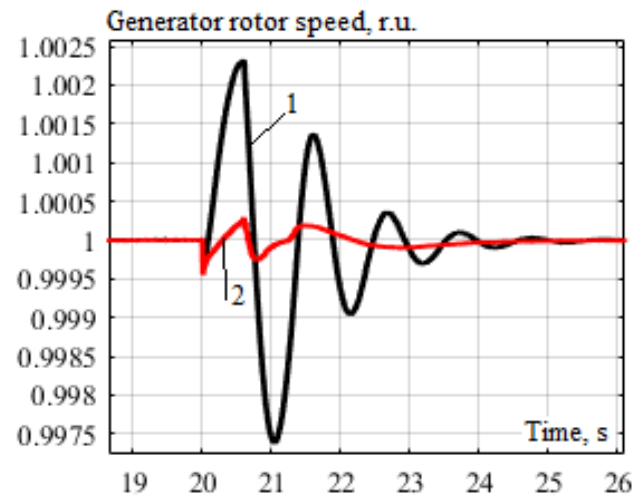

b)

Fig. 4. Time dependences of the turbine mechanical power (a) and the TGP generator rotor rotational speed (b) at a short-term short-circuit in the IPSS: 1 - the classic ASC (PID controller) was used; 2 - auto-prognostic ASC was used.

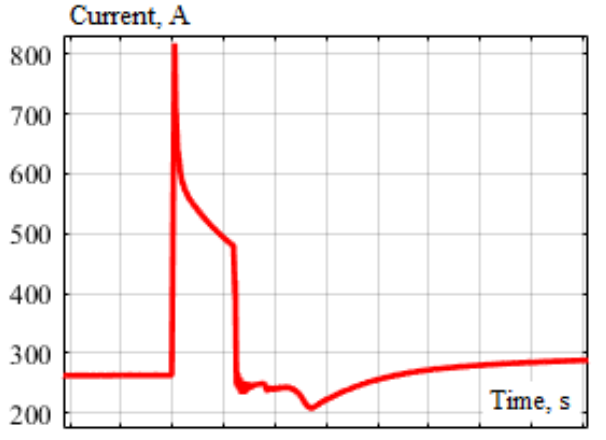

$\begin{array}{lllllllllll}19 & 19.5 & 20 & 20.5 & 21 & 21.5 & 22 & 22.5 & 23 & 23.5 & 24\end{array}$

a)

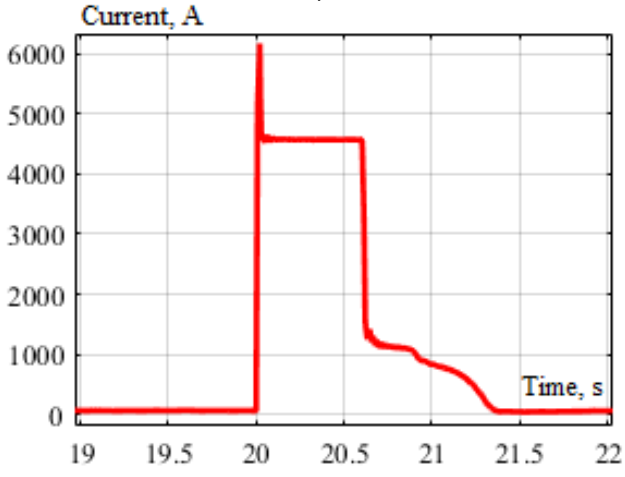

c)

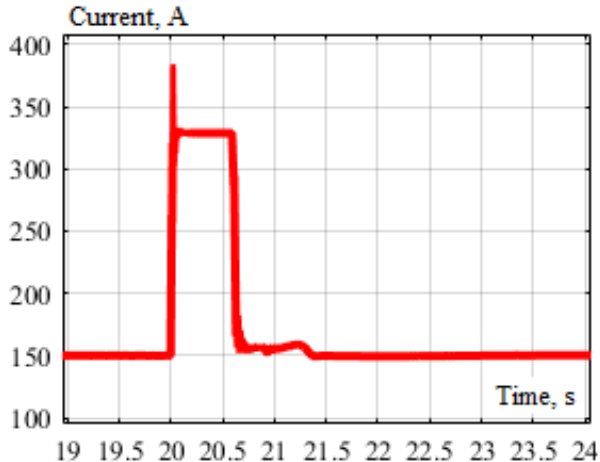

b)

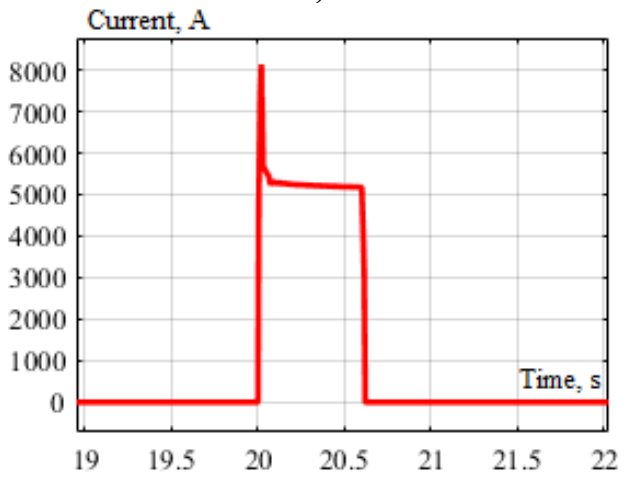

d)

Fig. 5. Time dependences of the effective values of the source currents in the IPSS under study at a three-phase short circuit: (a) - current of the TGP generator; (b) - current flowing from the WPP; (c) - current from ESU; (d) - total current at the short circuit location. 


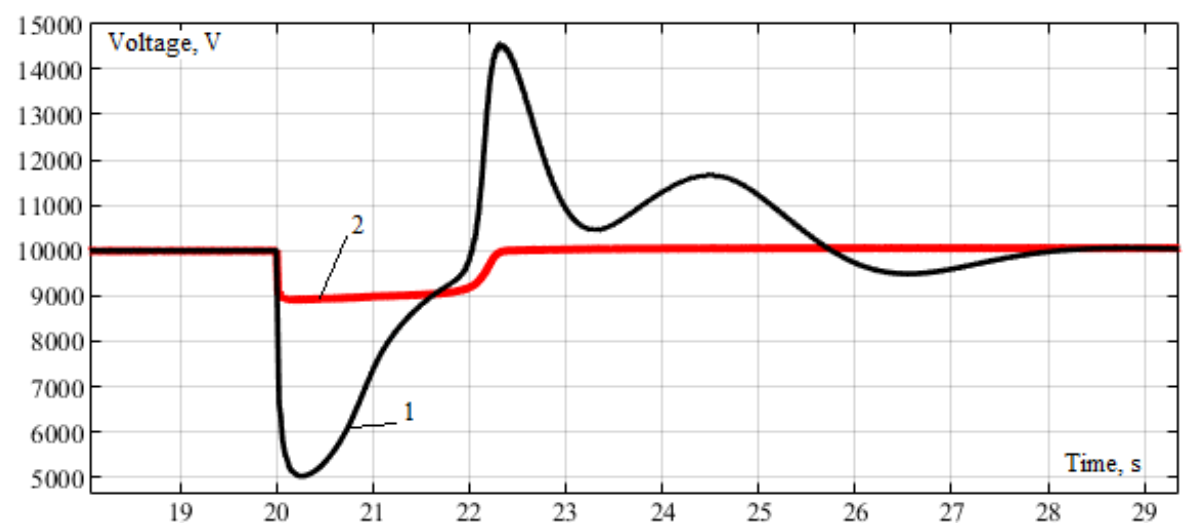

Fig. 6. The graph of the time dependence of the current voltage in the IPSS when starting a powerful equivalent AM: 1 - ESU is disabled; 2 - ESU is activated.

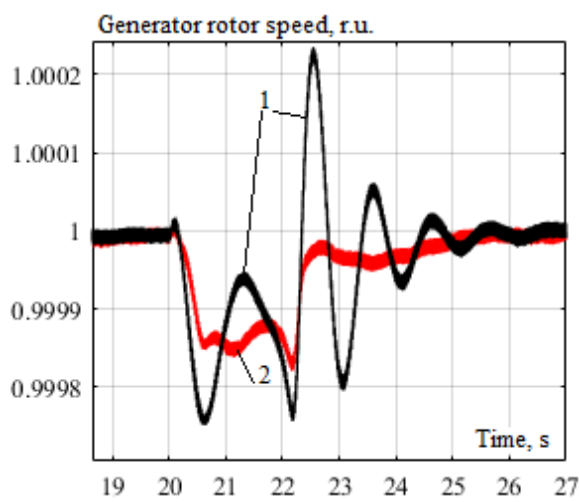

a)

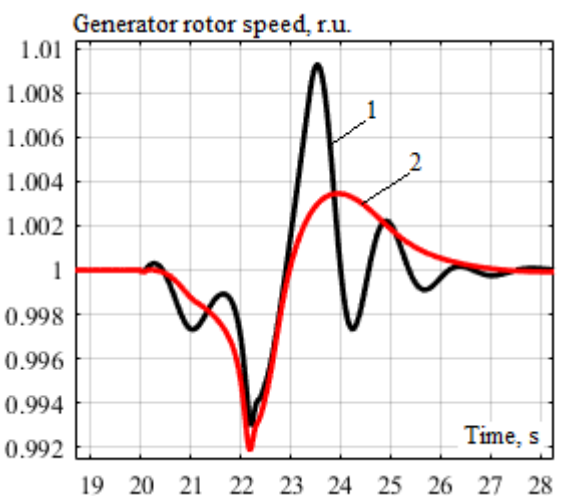

b)

Fig. 7. Time dependences of the TGP generator rotor speed when starting a powerful equivalent AM: a - complete IPSS diagram (ESU is activated); 6 - ESU is disabled; 1 - the classic ASC (PID controller) was used in TGP; 2 - TGP used auto-prognostic ASC.

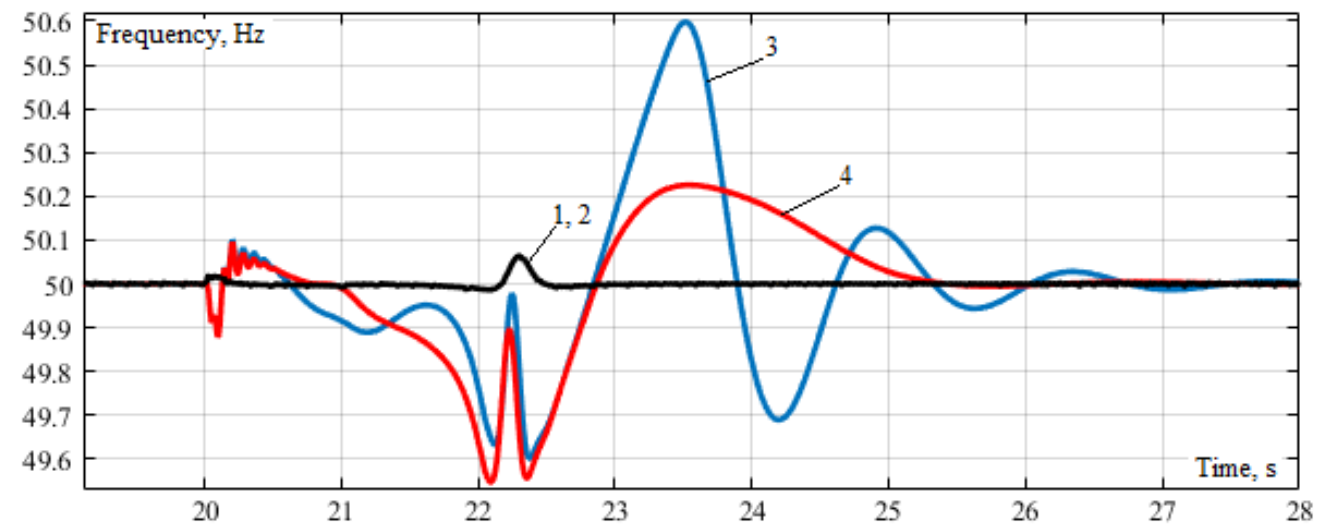

Fig. 8. The graph of the time dependence of the frequency in the IPSS when starting a powerful equivalent AM: 1 - ESU is activated, TGP auto-prognostic ASC was used; 2 - ESU is on, the TGP used the classic ASC (PID controller); 3 - ESU is disabled, the TGU used the classic ASC; 4 - ESU is disabled, TGP used auto-prognostic ASC.

in the IPSS requires checking the electric switchgear and revising the protection and automation setpoints.

Additionally, a direct start-up of an equivalent asynchronous motor with a power of $1485 \mathrm{~kW}$ was simulated as a disturbance, as a result of which a voltage dip is observed, and after starting, an overvoltage is observed. The use of a permanently connected powerful ESU in IPSS makes it possible to strictly limit the voltage deviation (Fig. 6): voltage dip is reduced by 5 times; there is no overvoltage when using ESU.

The use of auto-prognostic ASC in TGP also provided a positive effect when starting a powerful equivalent AM, consisting in a reducing of oscillation and overshoot value for the generator rotor speed (Fig. 7): the degree of damping of the rotor speed oscillations tended to unity; the overshoot value decreased on average by 2.5 times. It should be noted, 


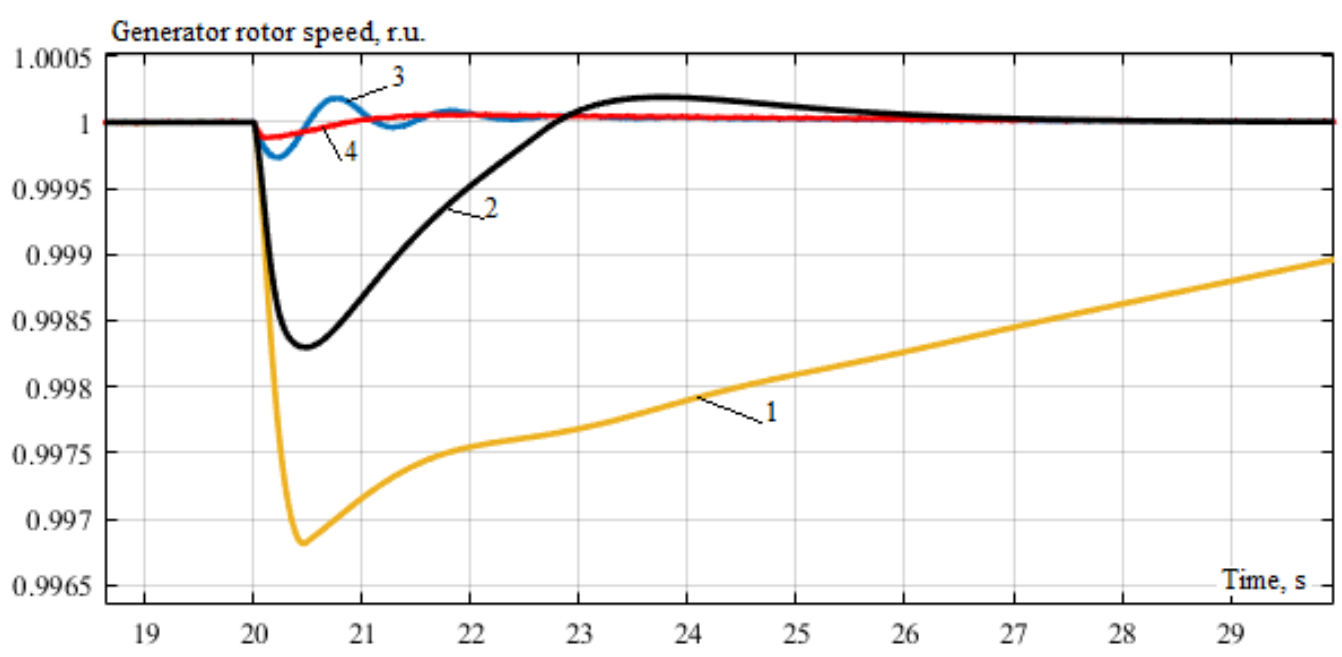

Fig. 9. The time dependences of the TGP generator rotor during emergency WPP shutdown: 1 - ESU is disabled, the TGU used the classic ASC; 2 - ESU is disabled, TGP used auto-prognostic ASC; 3 - ESU is on, the TGP used the classic ASC; 4 - ESU is activated, TGP auto-prognostic ASC was used.

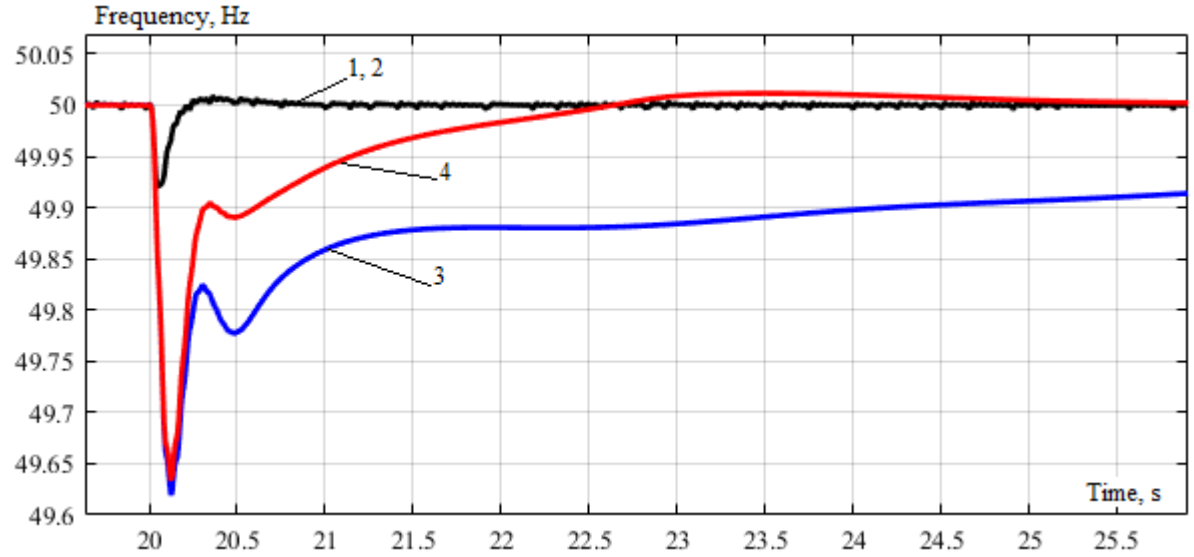

Fig. 10. The graph of the frequency time dependence in IPSS during emergency WPP shutdown: 1 - ESU is activated, TGP autoprognostic ASC was used; 2 - ESU is on, the TGP used the classic ASC; 3 - ESU is disabled, the TGU used the classic ASC; 4 ESU is disabled, TGP used auto-prognostic ASC.

that the use of high-power ESU and auto-prognostic ASC made it possible to significantly limit the deviation of the TGP generator rotor rotation speed: overshoot and oscillation were virtually absent (Fig. 7, a). Similar effects were observed for the network frequency as well (Fig. 8): the use of ESU strictly limited the frequency deviation; at the same time, the use of the autoprognostic ASC of TGP had no effect; with the ESU disabled, when the mode was maintained by the TGP and WPP, the use of the auto-prognostic ASC allowed to get rid of the oscillation and reduce the maximum frequency deviation by 3 times.

The results of modeling the emergency shutdown of WPP indicated that the use of a powerful ESU in the IPSS made it possible to significantly limit the voltage deviation, the rotational speed of the TGP generator rotor and the network frequency (Fig. 9-11); at the same time, the auto-prognostic ASC completely eliminated the oscillation and overshoot of the generator rotor speed (Fig. 9).

In the event of ESU disconnection, the autoprognostic ASC ensured the TGP generator stable operation during emergency shutdown of the WPP, as well as effective regulation of the rotor speed and frequency (Fig. 9 and 10).

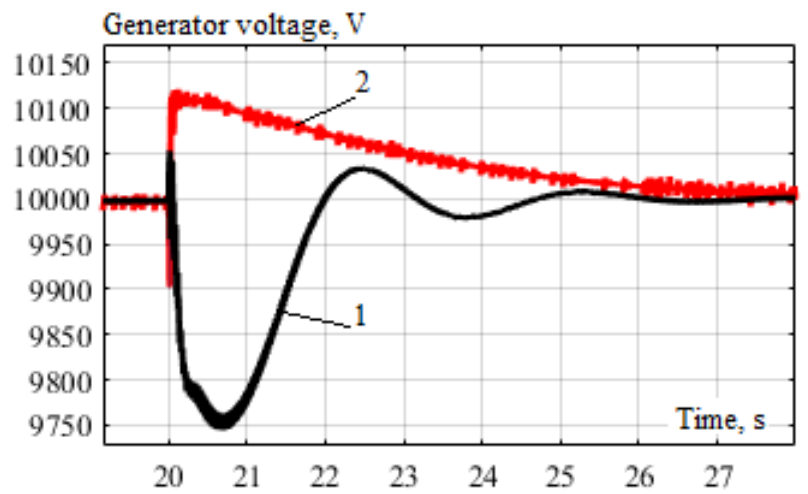

Fig. 11. The graph of the time dependence of the TGP generator voltage during emergency WPP shutdown: 1 - ESU is disabled; 2 - ESU is activated.

Thus, the performed modeling of the normal and emergency operating modes of the IPSS with 
asynchronous load indicated that the combined use of high-power ESU and the auto-prognostic speed controller of the TGP generator rotor makes it possible to ensure the stability and survivability of the IPSS, increasing the damping properties of the mode control system. The auto-prognostic ASC of the TGP generator does not require special adjustment of the regulator and allows to obtain high quality indicators of the rotor speed control and frequency in the IPSS network for all considered modes. The use of a fuzzy system to control the blades rotation angle of a wind power generation plant with an accumulator battery and a DC link ensures stable operation of the WPP in the normal and emergency modes under study.

\section{Conclusion}

The results of computer-based simulation of normal and emergency operating modes of an isolated power supply system with TGP, WPP and high-power ESU allow us to draw the following conclusions:

1. The auto-prognostic ASC of the TGP generator has a significant impact on the control processes in the case of a three-phase short circuit in the IPSS, making the turbine control more accurate and smooth. The overshoot and oscillation of the turbine mechanical power is significantly reduced, the time of the transient process for the rotational speed of the generator rotor is reduced by 1.8 times, and the overshoot value for the same parameter is reduced by 9 times.

2. The use of a permanently connected powerful ESU in IPSS makes it possible to strictly limit voltage deviations when starting a powerful asynchronous electric motor: voltage dip is reduced by 5 times; there is no overvoltage when using ESU.

3. The use of an auto-prognostic ASC when starting a powerful equivalent asynchronous motor reduces the oscillation and the overshoot value of the generator rotor speed. Combined use of high-power ESU and autoprognostic ASC allows to significantly limit the deviations of the TGP generator rotation speed. Similar effects are observed for the network frequency of an isolated power supply system.

4. In case of emergency shutdown of a WPP, the use of a powerful ESU allows to significantly limit the deviations of voltages, the TGP generator rotation speed and the network frequency; in this case, the autoprognostic ASC completely eliminates oscillation and overshoot. In case of ESU disconnection, the autoprognostic ASC ensures stable operation of the TGP generator and effective regulation of the rotor speed and frequency in the IPSS network.

5. The combined use of high-capacity ESU and autoprognostic controller of TGP generator speed allows to ensure stability and survivability of IPSS while enhancing its damping properties. The auto-prognostic ASC of the TGP generator does not require special adjustment and allows to obtain high quality indicators of the control and frequency for all considered modes.

6. The use of a fuzzy system to control the blades rotation angle of a wind power generation plant allows to ensure the WPP stable operation for all considered normal and emergency modes.

The research was carried out with financial support from a grant from the state assignment of the Ministry of Science and Higher Education of Russia (project No. 0667-2020-0039).

\section{References}

1. Y. Nishi, Journal of Power Sources, 100, 101-106 (2001)

2. P. Lombardi, Z.A. Styczynski, T. Sokolnikova, K. Suslov, Use of energy storage in isolated micro grids, Power Systems Computation Conference (PSCC), 1-6 (2014)

3. X. Shen, S. Zhu, J. Zheng, Y. Han, Q. Li, J. Nong, Sh. Mohammad, Active distribution network expansion planning integrated with centralized and distributed Energy Storage System, Power \& Energy Society General Meeting, Denver, CO, 1-5 (2015)

4. C. Savard, E.V. Iakovleva, A suggested improvement for small autonomous energy system reliability by reducing heat and excess charges, Batteries 5(1), 29 (2019)

5. A.A. Belsky, V.S. Dobush, D.I. Ivanchenko, D.Y. Gluhanich, Electrotechnical complex for autonomous power supply of oil leakage detection systems and stop valves drive control systems for pipelines in arctic region, Journal of Physics: Conference Series 1753(1), 012062 (2021)

6. N. Rugthaicharoencheep, S. Auchariyamet, International Journal of Electrical, Computer, Energetic, Electronic and Communication Engineering, 6, 385-389 (2012)

7. E.A. Martínez Ceseña, T. Capuder, P. Mancarella, IEEE Transaction on Smart Grid, 7, 348-357 (2016)

8. Ph.P. Barker, R.W. De Mello, Determining the Impact of Distributed Generation on Power Systems: Part 1 - Radial Distribution Systems, 2000 IEEE PES Summer Meeting, Seattle, WA, USA, 222-233 (11-15 July 2000)

9. N.I. Voropai, Z.A. Stychinsky, Renewable energy sources: theoretical foundations, technologies, technical characteristics, economics (Magdeburg: Otto-von-Guericke-Universität, 2010)

10. O. Ellabban, H. Abu-Rub, F. Blaabjerg, Renewable and Sustainable Energy Reviews, 39, 748-764 (2014)

11. B.M. Buchholz, Z.A. Styczynski, Smart Grids Fundamentals and Technologies in Electricity Networks (Springer-Verlag Berlin Heidelberg, 2014)

12. S.M. Magdi, M. AL-Sunni Fouad, Control and Optimization of Distributed Generation Systems (Cham: Springer International Publishing: Imprint: Springer, 2015) 
13. D. Guerra, E. Iakovleva, Mathematical modeling of parameters of solar modules for a solar power plant 2.5 MW in the climatic conditions of the Republic of Cuba, E3S Web of Conferences 140, 04013 (2019)

14. R. Wang, P. Wang, G. Xiao, Intelligent Microgrid Management and EV Control Under Uncertainties in Smart Grid (Springer, 2018)

15. Yu.N. Bulatov, A.V. Kryukov, Optimization of automatic regulator settings of the distributed generation plants on the basis of genetic algorithm, 2nd International Conference on Industrial Engineering, Applications and Manufacturing (2016)

16. A.V. Kryukov, S.K. Kargapol'cev, Yu.N. Bulatov, O.N. Skrypnik, B.F. Kuznetsov, Far East Journal of Electronics and Communications, 17, 1127-1140 (2017)

17. N.I. Voropai, P.V. Etingov, Two-Stage Adaptive Fuzzy PSS Application to Power Systems, Proc. of International Conference on Electrical Engineering ICEE'2001, Xi'an, China, 1, 314-318 (22-26 July 2001)

18. Yu.N. Bulatov, A.V. Kryukov, Neuro Fuzzy Control System for Distributed Generation Plants, Vth International workshop Critical infrastructures: Contingency management, Intelligent, Agent-based, Cloud computing and Cyber security (IWCI 2018), Atlantis Press, Advances in Intelligent Systems Research, 158, 13-19 (2018)

19. Yu.N. Bulatov, A.V. Kryukov, V.H. Nguyen, D.H. Tran, Advances in Intelligent Systems and Computing, 983, 105-115 (2019)

20. A.N. Skamyin, , I.V. Rastvorova, Consumed power regulation algorithm in the presence of high harmonics, Journal of Physics: Conference Series 1753(1),012042 (2021)

21. A. Udaratin, K. Loginov, A. Nemirovskiy, N. Rozhentsova, E. Gracheva, Modelling of emergency modes with FACTS devices installed, E3S Web of Conferences 178,01052 (2020)

22. E. Zamyatin, , I. Voytyuk, , E. Zamyatina, , Increasing the energy efficiency of an enterprise by point compensating of power quality distortions, E3S Web of Conferences, 140,04010 (2019)

23. Yu. V. Gulkov, , A.V. Turysheva, , P.B. Gurkin, , Electric power quality control in electro-technical complexes of oil processing plant, IOP Conference Series: Materials Science and Engineering 643(1),012001 (2019)

24. D.W. Clarke, Annual Review in Automatic Programming, 20, 83-94 (1996)

25. J.B. Rawlings, IEEE Control Systems Magazine, 38-52 (June 2000)

26. E.F. Camacho, C. Bordons, Model Predictive Control, 2nd edition (Springer, 2007)
27. G.A. Pikina, New in the Russian electric power industry, 3, 5-13 (2014)

28. Yu.N. Bulatov, A.V. Kryukov, V.H. Nguyen, Automatic prognostic regulators of distributed generators, International Multi-Conference on Industrial Engineering and Modern Technologies, Vladivostok: IEEE, 463, 1-4 (2018) 